\title{
Beitrag zur Lehre von der Lage correspondirender Netzhautpunkte.
}

\author{
Yon \\ Dr. Leop. Mandelstamm.
}

Bei der noch schwebenden Frage über die Identität de. beiden Netzhäute, $o b$ sie nämlich auf einer anatomisch praeformirten Anlage beruhe oder durch Erfahrung und Zweckmässigkeit vermittelst des Gesichtssinnes erworben sei, scheint es mir nicht überfü̈ssig einige Beobachtungen, welche ich auf diesem Gebiete im Laboratorium des Herrn Geheinmath Professor Helmholtz zu Berlin gewonnen habe, hier mitzutheilen. Die Versuche, welche ich über die Vergleichung der Länge correspondirender Netzhantstrecken, sowohl in horizontaler, als verticaler Richtung anstelite, sind nach dem Vorgange von Volkmann*) gemacht, unterscheiden sich jedoch sowohl durch ihe \&nordnung, als durch die ur. gleich grösseren der Vergleichung unterzogenen Distanzen. Bekanntich stellte Volkmann die Versuche fir verticale Distanzen in der Weise an, dass eine horizontale Linie ron 2 verticalen linien in dem Abstande dor

*) Physiolog. Unters, im Gebiete der Optik. Heft 2. Leipzig 1864. Helmholtz, Phys. Opt. pag. 706. 
Augen des Beobachters rechtwinklig geschnitten wurde, so dass jedes Auge ein rechtwinkliges + vor sich hatte. Unterhalb der Horizontalen nach aussen von den Verticalen befand sich eine zur erstereren parallele Linie, die eine feste Lage und beiläufig einen Abstand ron $5,5 \mathrm{Mm}$ hatte. Auf der anderen Seite war in eben der Weise eine andere, bewegliche Horizontallinie angebracht. Wurden nun bei parallelen Blicklinien beide Verticallinien zum Decken gebracht, so musste die bewegliche Horizontallinie so gestellt werden, dass sie mit der auf der anderen Seite befestigten eine continuirliche Gerade bildete. Darauf wurde die Lage der beiden Nadeln gewechselt und derselbe Versuch wiederholt. Das Mittel aus 30 Beobachtungen (einzelne Einstellungen wichen indessen bis $0,5 \mathrm{Mm}$. von der gegebenen Distanz ab) ergab eine derartig geringe Abweichung der zu vergleichenden verticalen Distanz mit der wirklichen, dass sie bei einer Entfernung (der Augen) des Beobachters von $30 \mathrm{Cm}$. kavm eine wahrnehmbare Grösse auf der Netzhaut bilikete. Ebenso verglich Volkmann auch horizontale Distanzen, indem er auf je einer rechten oder linken Seite der verticalen Linien in einer Distanz von 5,24 Mm. eine feste Verticale oberhalb und eine berregWiche unterhalb, oder umgekehrt anbrachte und sodann beide bei parallelen Bliclilinien in eine einzige Verticallinie zur Verschmelzung zu bringen suchte. Auch hier waren die Unterschiede der Mittelwerthe zu klein, um wahrgenommen werdea za können.

Ich stellte meine Versuche anfangs anch in ählicher Weise an, indem ich zwei Fäden im Abstande meiner Augen (62 Mm.) auf einer Wand mittelst zweier Gewichte herabhängen liess und nach links von dem rechten Faden eine Nadel in wechselnden Distanzen von 5-60 Mm. parallel zu jenem anklebte. Das Halbbild dieser Nadel suchte ich bei Festhaltung der mit parallelen Blicklinien 
zum Decken gebrachten Fäden mit dem einer anderu nach links vom linken Faden entgegengeführten Nadel zusammenzubringen. Diese Stelle wurde markirt und die Fntfernung rom linken Faden mit einem genauen Maassstabe gemessen. Es stellte sich aber dabei heraus, dass Differenzen von $0,5-10 \mathrm{Mm}$. in Bezug auf die gegebene Distanz auftraten, d. h. wenn ich die Deckung der beiden Fäden festzuhalten glaubte, konnte ich das einfach gesehene Bild der Nadeln bei forcirter Fixation allmälig weiter bis zu einèr ziemlichen, das ursprüngliche Maass überschreitenden Grenze vom linken Faden abrücken. Es konnte dies nur dadurch möglich geworden sein, dass die in horizontalen Distanzen zur Vereinigung gebrachten verticalen Fäden noch einfach gesehen wurden, während sie schon bereits in Doppelbildern erschienen waren, es also schwer war das Fusionsvermögen gänzlich zu eliminiren. *) Dagegen war es mir möglich genauere Resultate zu erhalten, weun ich statt der beiden Fäden zwei Nadeln vertical in demselben Abstande so anbrachte, dass die eine oberhalb, die andere unterhalb einer gedachten Horizontallinie sich befand, die Spitzen also bei paralleler Richtung der Blicklinien sich berührten. Hier war es zwar, namentlich bei etwas grösseren Distanzen schwieriger die Fixation der Nadelspitzen festzuhalten, wegen der Schwankungen der beiden Sehfelder; aber die Einstellungen waren darum auch genauer. Die Distanzen, die ich so gemessen, betrugen 5-60 Mm. Grössere Distanzen konnte ich bei einer Entfernung von $30 \mathrm{Cm}$. von der Wand, der Vergleichung nicht unterzichen. Zur Messung grösserer bediente ich mich eines gleichschenkligen rechtwinkligen Prisma's. Dasselbe wurde in der Höhe meiner Augen auf einen Tisch gestellt. An der mir gegenüberstehenden Wand befand sich in derselben

*) Cf. Helmholtz, Phys. Opt. pag. 707. 
Hohe und in verticaler Richtung eine ziemlich dicke Nadel. Seitlich vom Prisma, wie beifolgende Figur zeigt, stand ein Stativ mit einer solchen $\mathrm{Nadel}(\mathrm{a})$ und in einer ge-messenen Distanz yon dieser eine bremende Kerze (b). Wenn ich nun z. B. mit dem linken Auge die Narlel auf der Wand direct fixirte und mit dem rechten durch's Prisma blickte, so standen die beiden Nadelspitzen geraic ubereinander, vorausgesetzt dass die Strecke ao $=\mathrm{a}^{\prime} \mathrm{o}_{\text {, }}$ was anch der Fall ist, da die Kanto oc des Prisma's als Spiegel wirkt, welcher den Punkt a nach a' reflektirt; ebenso ist die Streeke $a b=a^{\prime} b$. Ausserdem sah ich

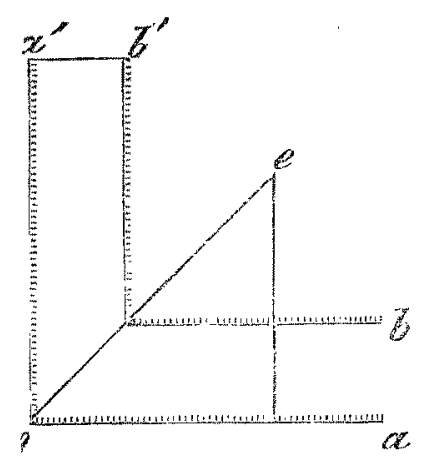
noch dureh das Prisma das an der Wand in derselben horizontalen Linie projicirte Bild der Plamme $b$ in $b^{\text {: }}$. Meine Aufgabe war es nun, bei festgehaitener Fixation der beiden Nadelspitzen, vermittelst des freien linken Anges einen duntrem schmalen Streifen Papiers mit der Flamme zum Decken zu bringen.

An der Wand war ein leinenes Millim.-Maass aufgeklebt, auf welchem sich die Entfernungen des Streifens von der Nadel an der Wand leicht ablesen liessen. Auf Minimaldifferenzen, wie in den Versuchen von Volkmann, konnte es hier unmöglich ankommen, zumal die Prifungsobjecte bei einer Distanz von $68 \mathrm{Cm}$. welche der Tisch hatte, eine erheblicho Ausdehnung erforderten. Es bandelte sich vielmehr darum festzustellen: wie weit die Fahigkeit der beiden Netzhauthälften (der äussern und innern) zur Wahrnehmung von Doppelbildern nach den Grenzen des Gesichtsfeldes hin reiche, resp. welcho Genauigkeit in der Unterscheidung einfacher von doppelten Bildern dort vorkomme. 
In gleicher Weise habe ich auch verticale Distanzen gemessen. Zu dem Zwecke habe ich zwei Nadeln so an der Wand befestigt, dass ihre Spitzen bei horizontalex Lage der Nadeln in der Entfernung meiner Augenmitten lagen. Unterhalb der einen Nadel habe ich parallel zu dieser in wechselnden Distanzen einen schmalen Streifen schwarzen Papiers aufgeklebt; unterhalb der andern Nadel war ein leinenes Milimetermaass befestigt. Es handelte sich darum, bei binocularer Vereinigung der beiden Nadelspitzen einen in der Hand gyehaltenen eben solchen Streifen Papiers mit jenem zum Decken zu bringen, so dass die oberen Ränder eine continuirliche Gerade bildeten. Die Lage 4er Streifen wurde alsdann vertauscht. Die Einstellungen geschahen hier sowohl für die oberhalb als unternalb der fixirten Nadelu liegenden Partien. Der Abstand meiner Angen von der Wand betrug auch hier $30 \mathrm{~cm}$., wobei der: Kopf, zur Vermeidung von Ungleichheiten der perspectitivischen Projection, durch ein eben so langes Brettchen an die Wand gestutzt var. Das jedesmalige Mittel ist aus 10 Einstellungen entnommen; die einzelnen Posten wurden addirt und die Durchschnittszahl mit + oder bezeichnet, je nachdem ein Zuwachs oder eine Abnahme zur gegebenen Distanz stattfand. Aus don einzelnen Beobachtungs- und den Durchschnittszahlen sind nach der Methode von Volkmann die whrscheinlichen Beobachtungsfehler berechnct worden. Die so gemessenen Distanzen erstreckten sich in horizontaler Bahu von 5-400 $\mathrm{Mm}$. in verticaler, unterhalb der eingehaltenen Fixation, bei Vergleichung der Declsstellen auf den oberen Netzhautpartien bis $200 \mathrm{Mm}$, bei oberhalb der Fixation ausgefühten Einstellungen, resp. der Prüfung der untern Netzhautregionen, bis $100 \mathrm{Mm}$., wegen dex, ungleich grössern Schwierigkeit des Erhennens im indirecten Sehen.

Beifolgende Tabelle möge die Verhälnisse erläutern: 
Mittel aus je 10 Einstellungen. Vergleichnng der Lage correspondirender Netzhautstrecken.

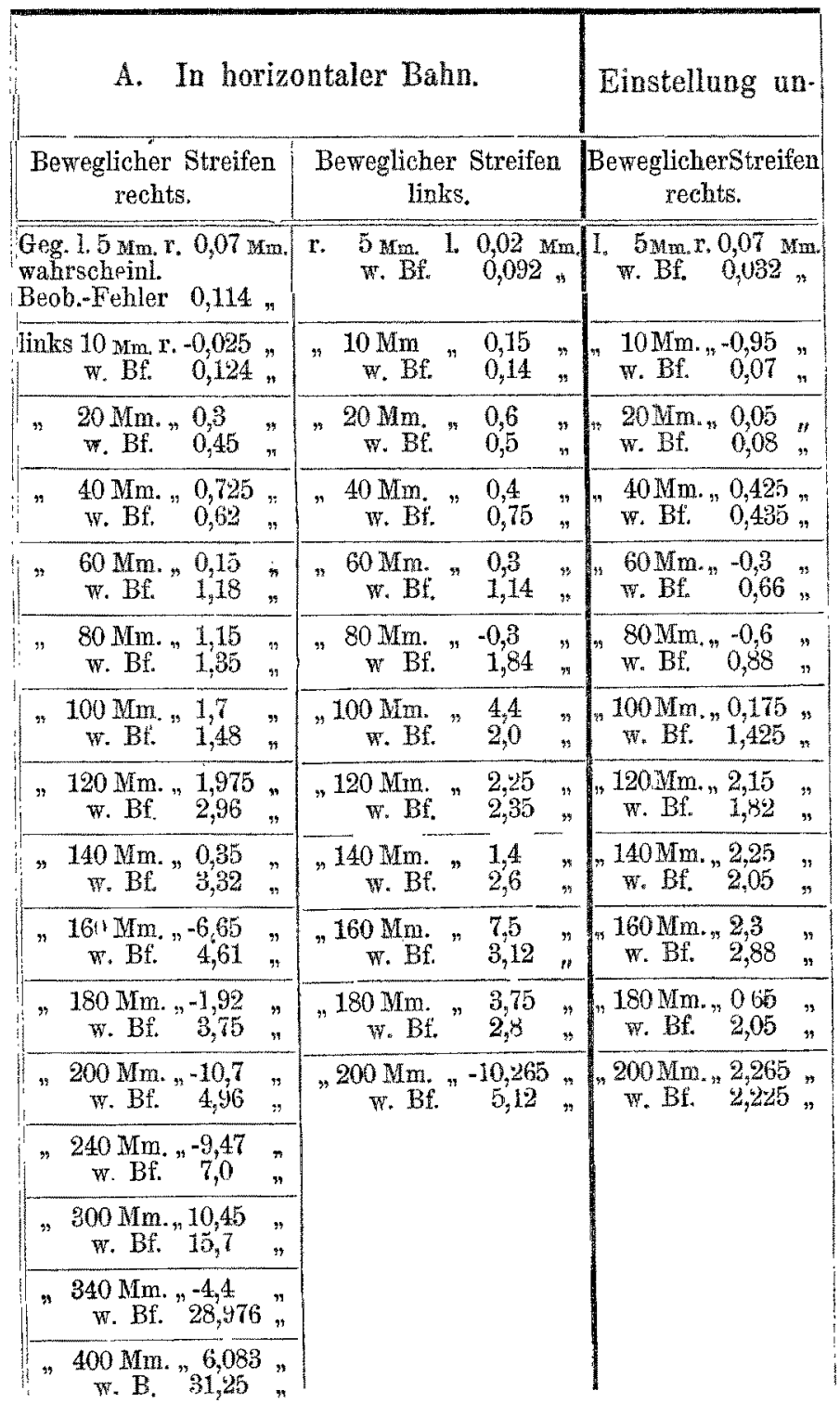


Mittel aus je 10 Einstellungen. Vergleichung der Lage correspondirender Netzhautstrecken.

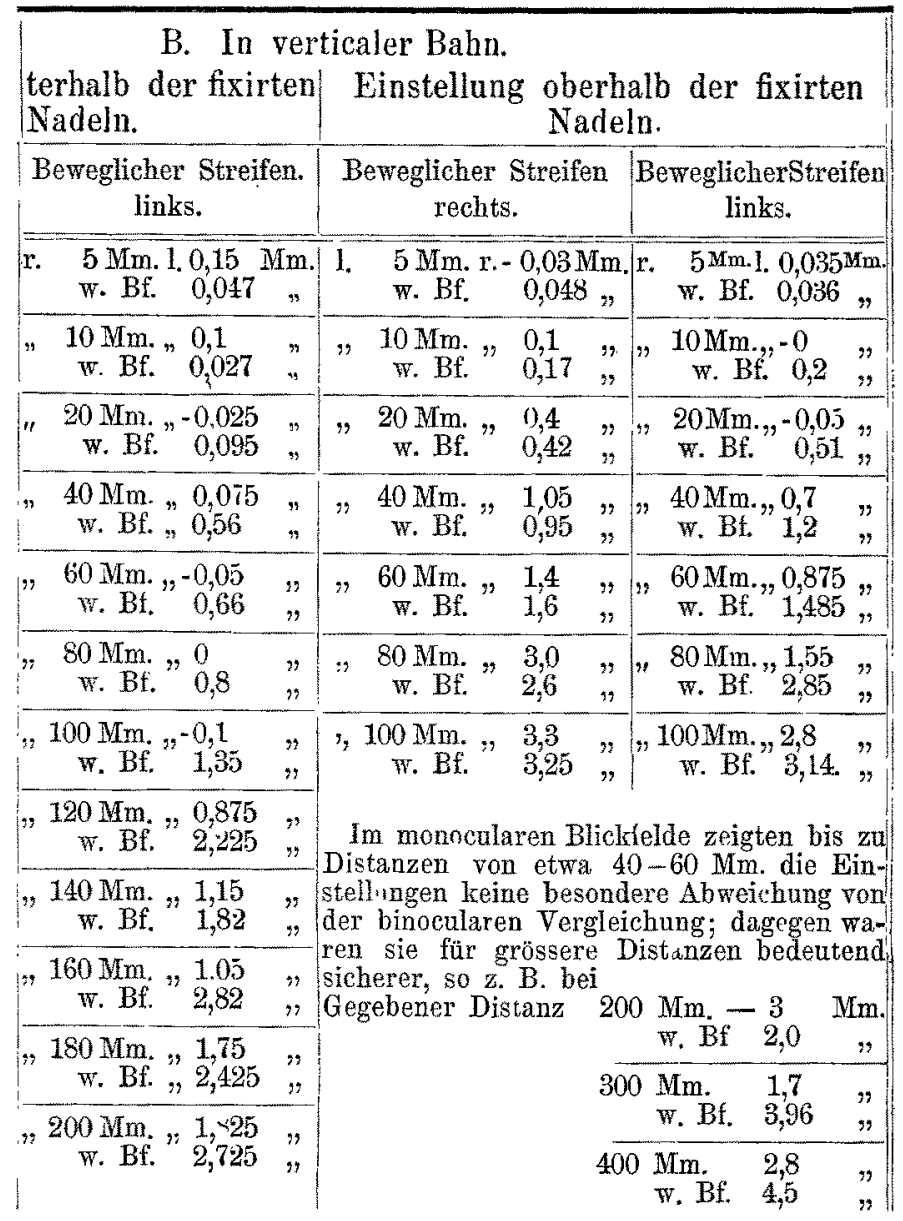


Aus der Tabelle ist zu ersehen, dass die Beobach. tungsfehler (resp. die Abweichnongen der einzelnen Einstellungen von der gegebenen Distanz) im indirecten Sehen zunehmen mit dem Wachsen der Entfernung des indirect gesehenen Gegenstandes vom Orte des fixirten Punktes (centralen Sehens). In verticaler Bahn sind für unterhalb der Fixation gelegene indirect gesehene Gegenstände die Einstellungen viel genauer, als caet. par. bei horizontaler. Denn dort haben wir erst bei $200 \mathrm{Mm}$. dieselbe Cngenanigkeit wie hier bei $100-120 \mathrm{Mm}$. Dagegen ist die Unsicherheit bei der Distanzvergleichnng indirect gesehener objecte in den untern Netzhauthäliften, bei oberhalb der Fixation stattgefundenen Einstellungen, am grössten, wie aus der Tabelle zu ersehen ist; denn beispielsweise eutspricht der Beobachtungsfehler von $100 \mathrm{Mm}$. hier haum $200 \mathrm{Mm}$. in ten oberen Netzhouthälften und etwa $140 \mathrm{Mm}$. in lorizontaler Richtung. Im monocularen Blickfelde hingegen sind die Wahrnehmungen der trennaren Objecte ziel deutlicher als bei binocularer Vergleichung. Fur kleinere Entfernungen ist kein besonderer Lnterschied zwischen beiden, dagegen nimmt die Genauigkeit der ersteren bei immer wachsenden peripherischen Strecken im Vergleich zur letzteren bedentend zu, so dass sie nur $1 / 2,1 / 3,1 / 8$ der Ungenaugkeit dieser ausmacht.

Diese Versuche sind daza angethan einen kleinen Beitrag fur die Ansicht zu liefern, dass die Fähigheit unserer Netzhäuto gesonderte Eindrucke in einen zu verschmelzen, lediglich von dem zweckmässigen Gebrauch unserer Angen, resp. dem Urtheil und der Frfahrung abhängt. Die identischen Punkte als solch wïrden nur in diesem Sinne existiren und nicht als anatonisch präformirte Gebilde. So wie wir für gewöhnlich, namentlich mit etwas gesenkter Blickebene einherzugehen ptlegen und meist auf die Gegenstande, die vor und unter uns 
liegen mehr, als auf die in eben denselben Entfernungen seitlich liegenden unsere Aufmerlssamkeit richten, dagegen höher gelegene Gegenstünde relativ nur selten beobachten, so sehen wir auch die harmonische Zusammenwirkung in den oberen Partien der Netzhäute am stärksten ausgebildet, weniger in den horizontalen und am wenigsten in den unteren Regionen. Ferner sehen wir in den äussersten Zonen der Netzhäute die Identität bei weitem nicht so hergestellt, wie in dem gewöhnlichsten Orte des deutlichen Sehens, der Mac. lut. näher gelegenen, was eben darauf hindeutet, dass in diesen Partien, die beim gewöhnlichen Sehen so wenig in Anspruch genommen werden, noch ?ein einheitliches Verhältniss durch unsere Gewohnheit und Erfahrung sich herausgebildet hat. Das Gesetz des Einfachsehens ist ubrigens an die Identität der Netzhäute nicht streng gebunden, ebenso wie nicht identische Netzhautstellen einen einfachen Eindruck bedingen können. Ich erinnere nur an die Fälle von concom. Schielen und rückgängigen Paralysen. Im Uebrigen verweise ich in Butreft gerade dieses Abschnittes auf die Arbeiten von Helmholtz, Nagel, Wundt etc. 